\title{
Development of Segmentation-Rendering on Virtual Reality for Training Deep-learning, Simulating Landscapes and Advanced User Experience
}

\author{
Tomohiro Fukuda ${ }^{1}$, Marcos Novak ${ }^{2}$, Hiroyuki Fujii ${ }^{3}$ \\ ${ }^{1}$ Osaka University ${ }^{2}$ University of California Santa Barbara ${ }^{3}$ Forum8 Co., Ltd. \\ Ifukuda@see.eng.osaka-u.ac.jp ${ }^{2}$ marcos@mat.ucsb.edu3fujii@forum8.co. \\ jp
}

\begin{abstract}
Virtual reality (VR) has been suggested for various purposes in the field of architecture, engineering, and construction (AEC). This research explores new roles for VR toward the super-smart society in the near future. In particular, we propose to develop post-processing rendering, segmentation-rendering and shadow-casting rendering algorithms for novel VR expressions to enable more versatile approaches than the normal photorealistic red, green, and blue (RGB) expressions. We succeeded in applying a wide variety of $V R$ renderings in urban-design projects after implementation. The developed system can create images in real time to train deep-learning algorithms, can also be applied to landscape analysis and contribute to advanced user experience.
\end{abstract}

Keywords: Super-smart society, Virtual Reality, Segmentation, Deep-learning, Landscape simulation, Shader

\section{INTRODUCTION}

The so-called "super-smart society" is approaching in the near future through such innovations as industry 4.0 and society $5.0[1,2]$. In this society, a huge amount of information (big data) from sensors and the Internet of Things devices in physical space will be accumulated in virtual space. Artificial intelligence will analyze this data, and the results will be fed back to humans in physical space in various forms. Virtual reality (VR), which has features of presence, interaction, and autonomy (Zeltzer, 1992), and was selected one of the 14 Grand Challenges for Engineering in the 21 st Century [3], has been suggested for various purposes such as design study, presentation, simulation and communication in the field of architecture, engineering, and construction (AEC) (Dokonal et al. 2016, Dorta et al. 2016, Natephra et al. 2017, Eloy et al. 2018, Fukuda et al. 2019).

This research explores new roles for VR in the super-smart society. In particular, we propose to develop post-processing rendering, segmentationrendering, and shadow-casting rendering algorithms for novel VR expressions to enable more versatile approaches for training deep-learning, simulating landscapes and advanced user experience than the photorealistic red, green, and blue (RGB) expressions that are normally used in VR. 


\section{SITUATIONS AND PROBLEMS}

Toward the realization of super-smart society, the situations and problems examined in this research are as follows:

Self-driving cars and smart-inspection, which are projected to be outputs of the super-smart society, are inseparable from AEC fields. In order to realize these technologies, various kinds of training must be performed on self-driving/smart-inspection systems (Kuwata et al. 2009, Yabuki et al. 2018). Training using physical space and physical objects is limited in terms of the various types that can be prepared, and costs tend to become high. By contrast, training using virtual space and virtual objects readily increases the types of training data that can be used and tends to reduce cost.

Deep-learning has become a popular subset of machine learning due to its high level of performance and applied to the AEC field (Cao et al. 2019). A great way to use deep-learning to segment objects in images is to build a convolutional neural network (CNN). This CNN needs a large amount of training data (images in this study) for learning. In fact, photographs are often used, but it is not easy to collect large amounts of photographs. For learning, in addition to a normal RGB image, its paired foreground/background (binary) and parts-based (categorical) shape images of it are also required. Using a 3D virtual model in VR makes it easy to change parameters such as camera information and objects' properties, thereby making it possible to readily and automatically create a large amount of image data for learning if the 3D model's accuracy is high. On the other hand, a system for simply generating foreground/background (binary) and parts-based (categorical) shape images is needed (Problem-A).

Via the concept of a "digital twin," which is closely related to the super-smart society, digital copies of physical buildings and urban spaces can be used for real-time optimization (Söderberg et al. 2017). VR is then expected to act as an interface that presents information to users in physical space via an easy-tounderstand method with various expressions during feedback. When simulating landscapes in architectural and urban-design process, design targets are normally studied in the VR space expressed in photorealistic RGB. On the other hand, it is easier to understand by visualizing according to analytical targets such as the distance from the main viewpoint to the design target (visual distance: short-distance view, middle-distance view, long-distant view) and the shadow-falling duration per point (Problem-B).

\section{PROPOSED VR SYSTEM}

In order to tackle the problems described in Chapter 2, we develop post-processing rendering, segmentation-rendering, and shadow-casting rendering algorithms. Regarding segmentationrendering method, VR images can be divided into regions and categories corresponding to different objects or parts of objects in real-time.

We developed new rendering functions on UCwin/Road, which is a VR software for which rendering customization function is provided (Figure 1 (a)). Generally, it can execute rendering processing on the screen multiple times, which is usually only once. It can also implement pre-processing and post-processing of the whole rendering, and preprocessing and post-processing of one scene rendering respectively. Furthermore, it can define variable values to be delivered to the shader when rendering objects, which can be used on the shader processing side. These functions can be implemented as plug-in software and can be switched on VR (Figure 2).

In order to realize a segmentation algorithm for each type of 3D object constituting a VR application, the types of 3D objects are divided into Road, River, Intersection, Model, RoadSideModel, Character, Car, Sign, and so on. In these types, Road is further subdivided into Guardrail, Road, Bridge, Tunnel, Sidewalk, etc., as a lower hierarchy. Similarly, Model is further comprised of Vehicle, Building, Structure, TrafficLight, Plant, etc. as a lower hierarchy (Figure 1 (b)). 
Figure 1

(a) Rendering customization feature: The general processing flow, (b) Object definition for Segmentation

Figure 2 Switching rendering options on VR

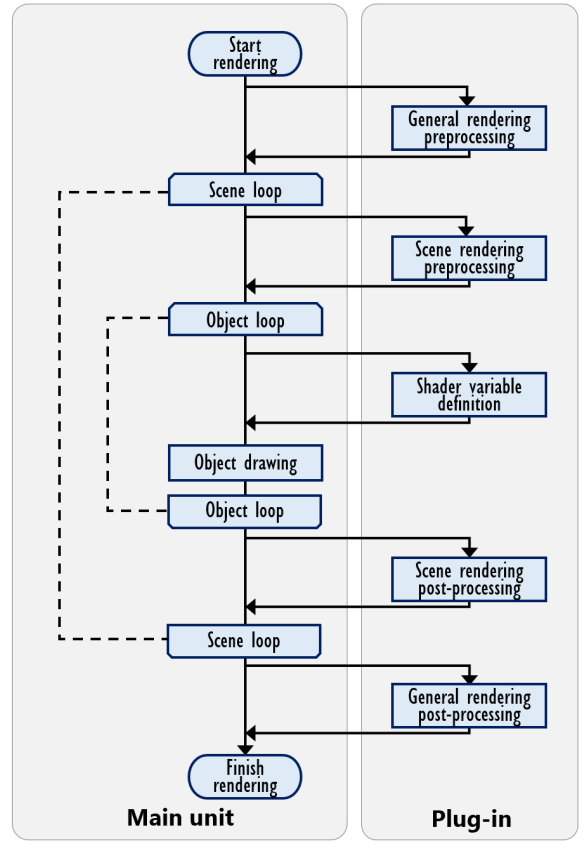

(a)

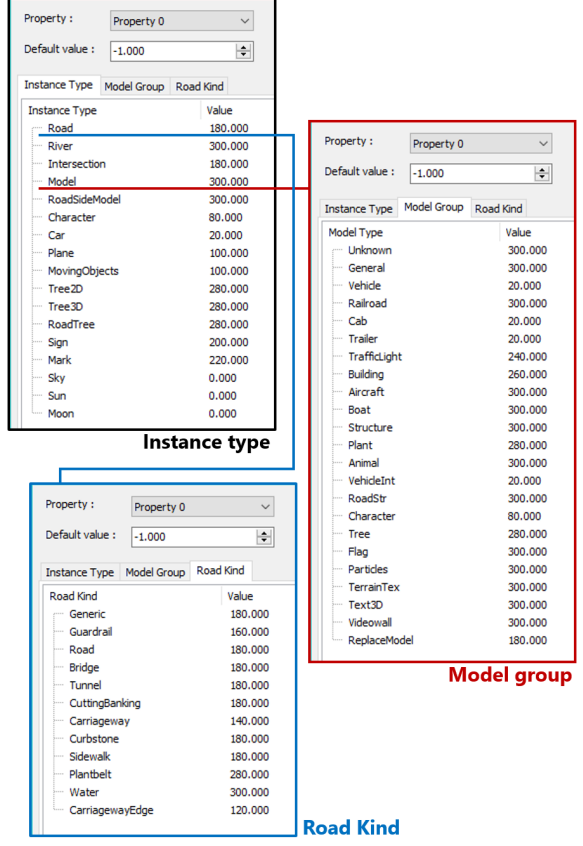

(b)

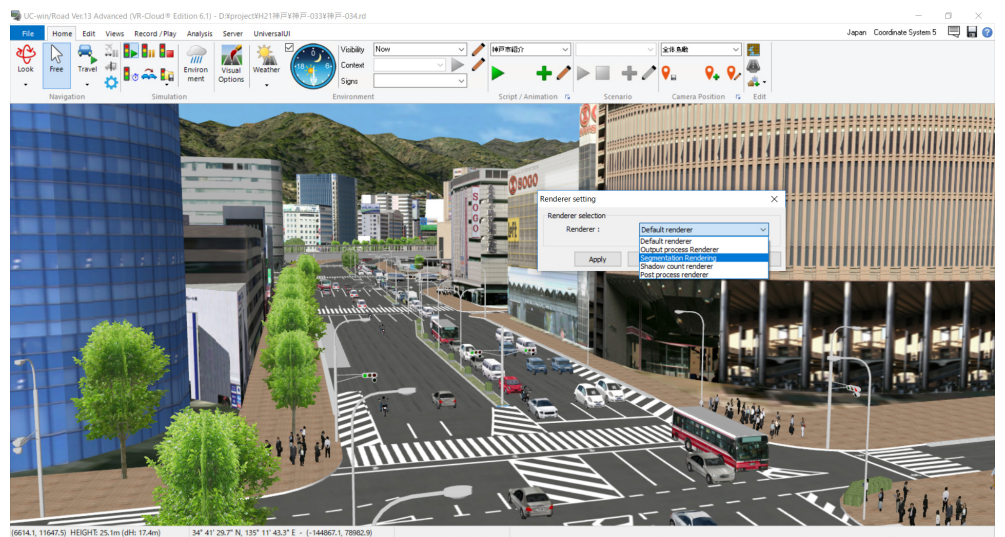




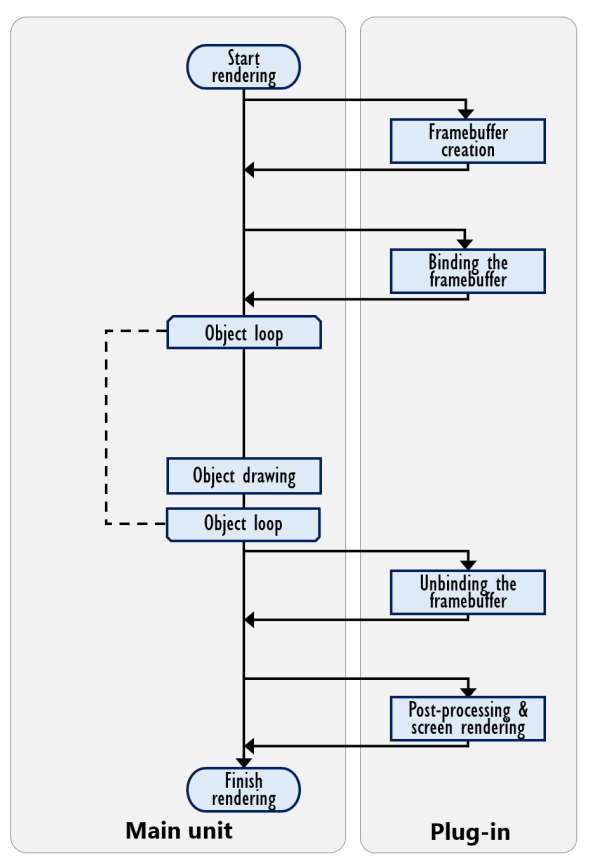

(a)

\section{Post-processing rendering}

First, we implemented post processing as the simplest rendering customization processing (Figure 3 ). In the pre-processing, rendering is performed in a framebuffer which is a temporary rendering destination, and post-processing is performed from the rendering result and output to the screen. The shader program is coded by GLSL (OpenGL Shading Language) and can be customized by the user.

\section{Segmentation rendering}

We implemented a plug-in that segments objects and colors each object to generate materials for various machine learning and for landscape analysis (Figure $4(\mathrm{a}))$.

In pre-processing of the entire rendering, create a framebuffer to be a temporary rendering destination, and apply a shader programmed to write color

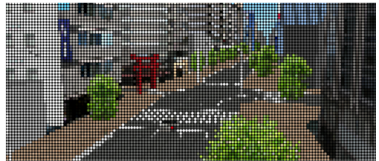

(b)

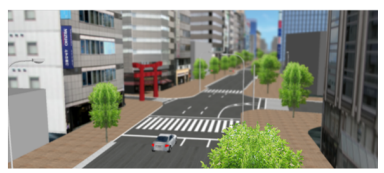

(c)

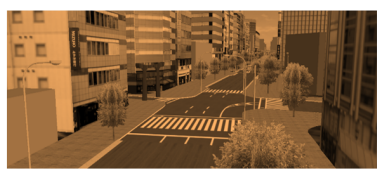

(d)

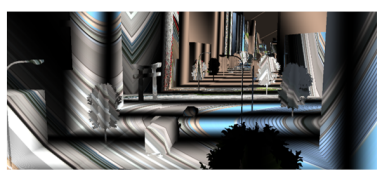

(e)
Figure 3

Post-processing: (a)

Whole processing

flow, (b) Ball, (c)

Focus, (d)

Monochrome, (e)

Artistic information, normal information, depth information, and property values of the scene to five textures. Next, in the object drawing process, the hue value according to the type of the objects, speed information, acceleration information, distance from the viewpoint, height information from the terrain is calculated and delivered as the property values on the plug-in. We implemented the processing to calculate the final output using the rendered texture in postprocessing of the entire rendering. According to this implementation, it is possible to switch and execute segmented coloring, speed, acceleration, distance from the viewpoint, and height from the ground from ordinary RGB representation by changing the setting of post-processing.

The internal-rendering flow of the proposed segmentation rendering is described as follows (Figure 4 (b)). The input data (matrices, vertices, normals, and 
Figure 4

Segmentation rendering: (a) Whole processing flow, (b) Rendering flow

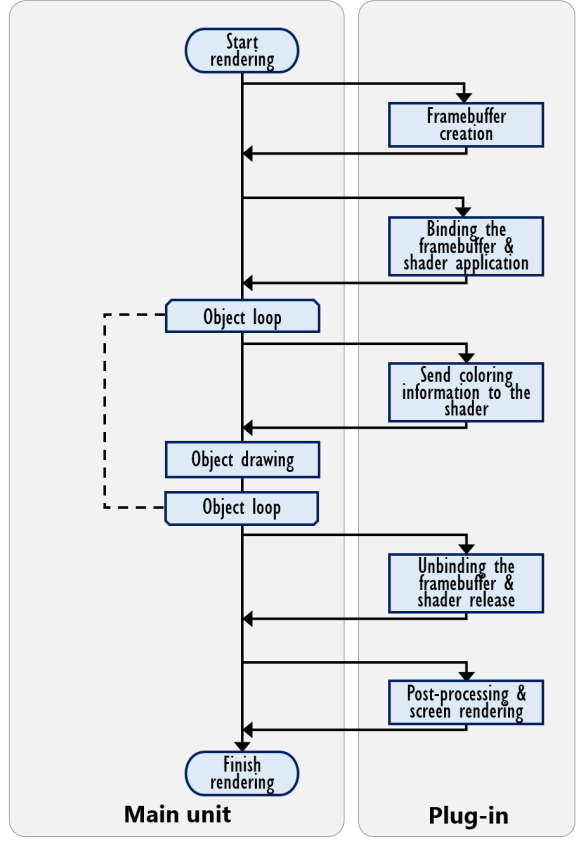

(a)

property values) are sent to the GPU (Graphics Processing Unit). In the first texture-rendering phase, the vertex-shader process consists of calculating screen positions and normals and then calculating color by vertices. The fragment-shader process consists of obtaining texture color, multiplying the color calculated in the vertex shader, and then outputting the color, depth, normal, and property values to screen-space textures. In the next segmentation mixing phase, the vertex-shader process consists of outputting quad of the screen. The fragment-shader process consists of obtaining colors, normals, depths, and property values from screen-space textures and then setting pixel colors based on these values.

\section{Shadow-casting rendering}

In the VR city model, we implemented multishadowing as a plug-in software to simulate the ef-

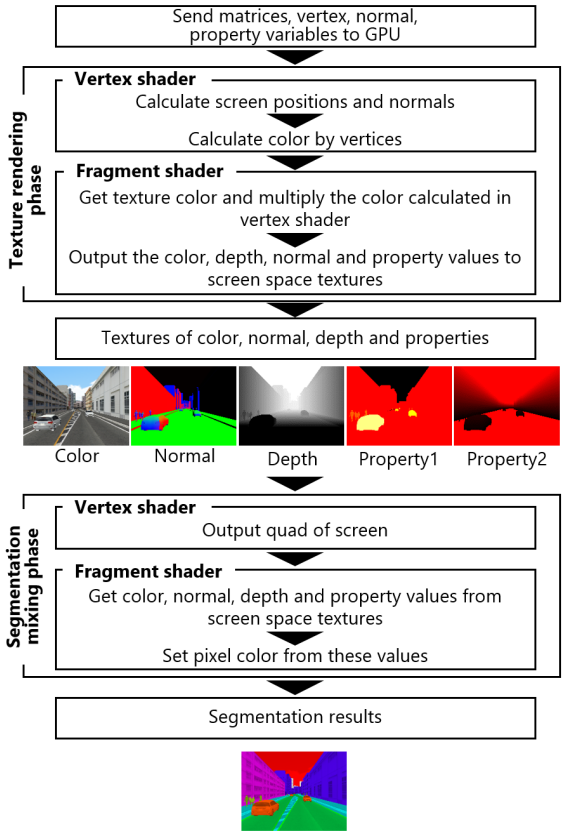

(b)

fects of shadow-falling a day (Figure 5 (a)).

In normal shadow rendering processing, the scene color and the shadow color are mixed and displayed. On the other hand, in the proposed system, the scene color is not used, and the part where the shadow falls is black and the part where the sun shines is white (Figure 5 (b)). Next, time-varying scenes are rendered multiple times and their rendered results are blended. Then, it was rendered that the part with many suns is white and the part with many shadows is black, and it was temporarily written in the framebuffer (Figure 5 (c)). Finally, the calculation results are colored and output to the screen as a heat map in order to clearly show the effects of sunshine in the post-processing (Figure 5 (d)). 


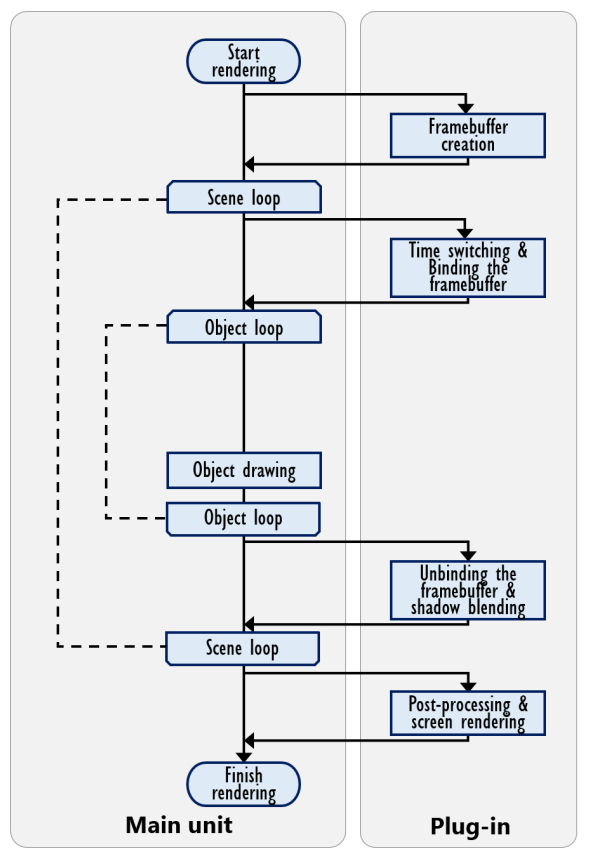

(a)

\section{RESULTS AND DISCUSSION}

We applied the developed system to VR urbandesign applications and realized the following wide variety of renderings (Figure 6). Various VR expressions with real-time rendering and user interaction were made possible based on the large-scale 3D-VR model.

Post-processing rendering is output by shaders as a set of ball-like expressions, focused expressions, monochrome expressions, posterization expressions, artistic expressions, etc., which will contribute to the advanced user experience.

Object Segmentation involves segmentation by each type of 3D object described in Chapter 3 (for Problems A and B). Using this VR rendering method, foreground/background (binary) and partsbased (categorical) shape images can be easily created by changing the camera viewpoint (automation

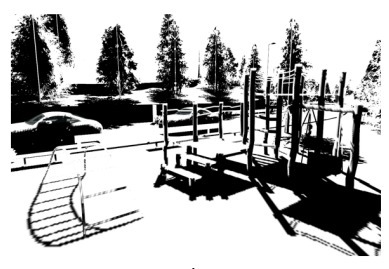

(b)

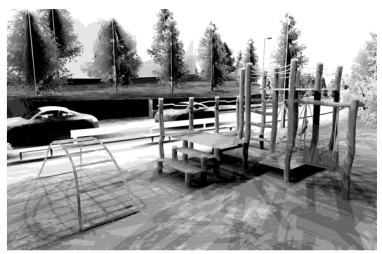

(c)

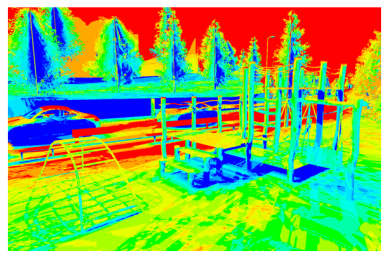

(d)
Figure 5

Shadowing: (a)

Whole processing

flow, (b) One

shadow rendering, (c) Blended image with multiple shadow rendering results, (d) Heat map by post-processing is possible). $R G B+$ Object Segmentation is expressed by mixing normal photorealistic RGB and segmentation representations based on the types of $3 \mathrm{D}$ objects described in Chapter 3 (for Problems A and B). A user can experience VR while simultaneously looking at the appearance of the object and its type.

Velocity Segmentation involves segmentation according to the speeds of moving 3D objects such as cars and pedestrians (for Problems A and B). Acceleration Segmentation involves segmentation according to the acceleration of moving 3D objects such as cars and pedestrians (for Problems $A$ and $B$ ). In normal RGB expression, it is difficult to understand the velocity and acceleration of moving objects such as cars and pedestrians. On the other hand, this VR expression makes it possible to intuitively understand the velocity and acceleration of moving objects.

Distance Segmentation involves segmentation 
Figure 6

Developed wide variety of renderings on VR
Photorealistic rendering on VR

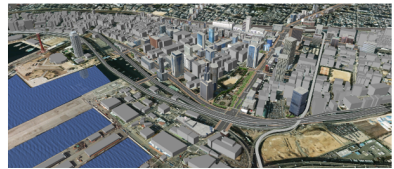

Birds-eye view

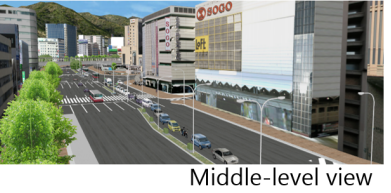

Middle-level view

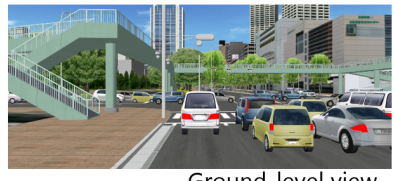

Ground-level view

Real-time rendering, User interaction

Post-processing rendering on VR
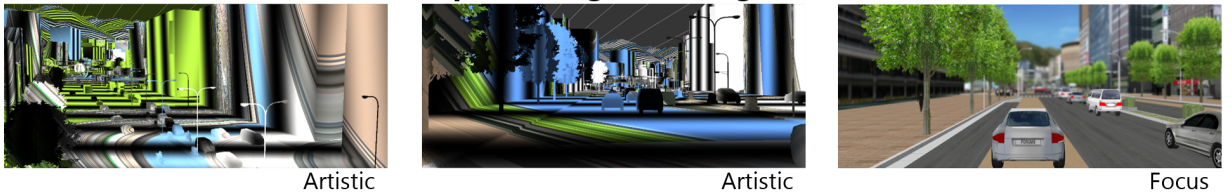

\section{Segmentation rendering on VR}
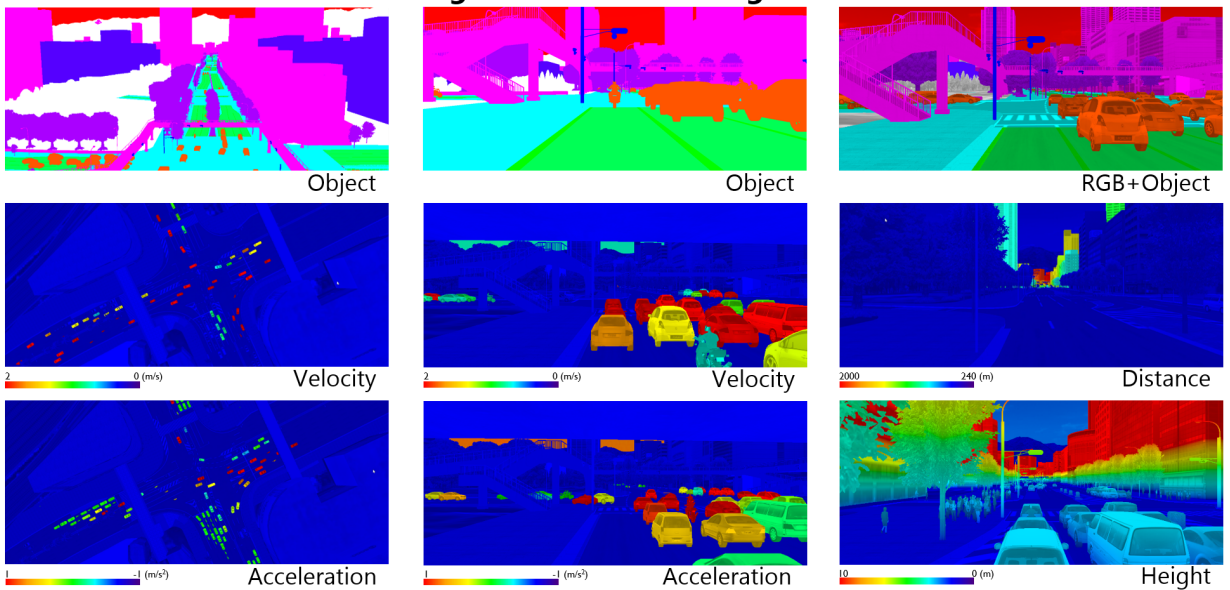

Shadow rendering on VR
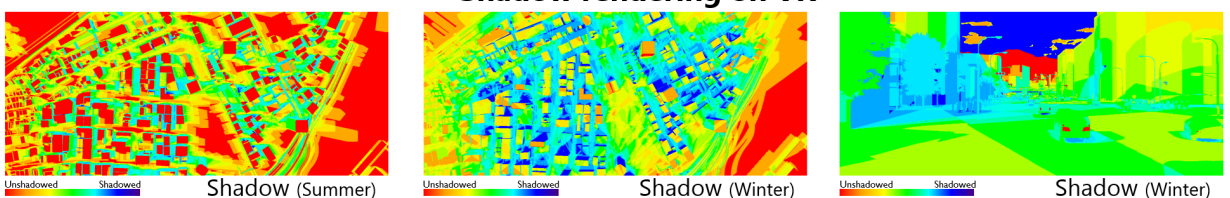

according to the distance from the VR camera, which is the viewpoint of pedestrians and drivers who operate VR (for Problems A and B). Height Segmentation involves segmentation according to the height from the ground level of the terrain (for Problem B). When considering landscape design, it is important to understand the distance from the viewpoints of each element that consists of the urban landscape. The ele- 
ments that compose the landscape look different as short-distance view, middle-distance view, and longdistance view depending on the distance from the viewpoint. The intuitive examination has been difficult in the conventional photorealistic rendering. On the other hand, this VR expression makes it possible to intuitively understand the distance from the viewpoint to each element and the height from the ground surface.

Shadow-count rendering involves expression according to shadowing duration to each point in the VR space at summer and winter solstices, respectively (for Problem B). This VR expression is a tool that can perform more sophisticated shadow simulation and can support more detailed design.

\section{CONCLUSIONS}

This research contributes to the development of post-processing rendering, segmentation-rendering and shadow-casting rendering algorithms for novel VR expressions by presenting situations and problems related to the super-smart society. We succeeded in applying a wide variety of rendering outputs to VR applications of urban-design projects. The developed system can easily create foreground/background (binary) and parts-based (categorical) shape images in addition to a normal photorealistic RGB image to train deep-learning algorithms, can also be applied to landscape simulation according to analysis targets such as the distance from the main viewpoint to the design targets and the shadow-falling duration per point in VR urban space, and contribute to advanced user experience.

\section{ACKNOWLEDGMENTS}

This research was done with one of the research activities from 2017 to 2018 by World16 (Yoshihiro Kobayashi, Kostas Terzidis, Marc Aurel Schnabel, Paolo Fiamma, Amar Bennadji, Thomas Tucker, Dongsoo Choi, Matthew Swarts, Ruth Ron, Taro Narahara, Wael Abdelhameed, Marcos Novak, and Tomohiro Fukuda), an international research group on virtual reality. The authors wish to acknowledge the support received from Forum8 Co. Ltd.

\section{REFERENCES}

Cao, R, Fukuda, T and Yabuki, N 2019 'Quantifying Visual Environment by Semantic Segmentation Using Deep Learning', Proceedings of CAADRIA 2019, Wellington, pp. 71-80, Vol.2

Dokonal, W, Knight, M and Dengg, E 2016 'VR or Not VR No Longer a Question?', Proceedings of eCAADe 2016, Oulu, pp. 573-579, Vol.2

Dorta, T, Kinayoglu, G and Hoffmann, M 2016, 'Hyve3D and the 3D Cursor: Architectural co-design with freedom in Virtual Reality', International Journal of Architectural Computing, 14(2), pp. 87-102

Eloy, S, Ourique, L, Woessner, U, Kieferle, J and Schotte, W 2018 'How Present am I - Three virtual reality facilities testing the fear of falling', Proceedings of eCAADe 2018, Lodz, pp. 717-726, Vol.2

Fukuda, T, Yokoi, K, Yabuki, N and Motamedi, A 2019, 'An Indoor Thermal Environment Design System for Renovation Using Augmented Reality', Journal of Computational Design and Engineering, 6(2), p. 179-188

Kuwata, Y, Teo, J, Fiore, G, Karaman, S, Frazzoli, E and How, JP 2009, 'Real-Time Motion Planning with Applications to Autonomous Urban Driving,', IEEE Transactions on Control Systems Technology, 17(5), pp. 1105-1118

Natephra, W, Motamedi, A, Fukuda, T and Yabuki, N 2017, 'Integrating building information modeling and virtual reality development engines for building indoor lighting design', Visualization in Engineering, pp. 5-19

Söderberg, R, Wärmefjord, K, Carlson, JS and Lindkvist, L 2017, 'Toward a Digital Twin for real-time geometry assurance in individualized production', CIRP Annals, 66(1), pp. 137-140

Yabuki, N, Nishimura, N and Fukuda, T 2018 'Automatic Object Detection from Digital Images by Deep Learning with Transfer Learning,' Advanced Computing Strategies for Engineering. EG-ICE 2018 (25th Workshop of the European Group for Intelligent Computing in Engineering). Lecture Notes in Computer Science, Lausanne, pp. 3-15, Vol.10863

Zeltzer, D 1992 'Autonomy, Interaction, and Presence', Presence, pp. 127-132, Vol.1

[1] http://alvarestech.com/temp/tcn/CyberPhysicalSyst ems-Industrial4-0.pdf

[2] https://www8.cao.go.jp/cstp/english/society5_0/in dex.html

[3] http://www.engineeringchallenges.org/challenges. aspx 